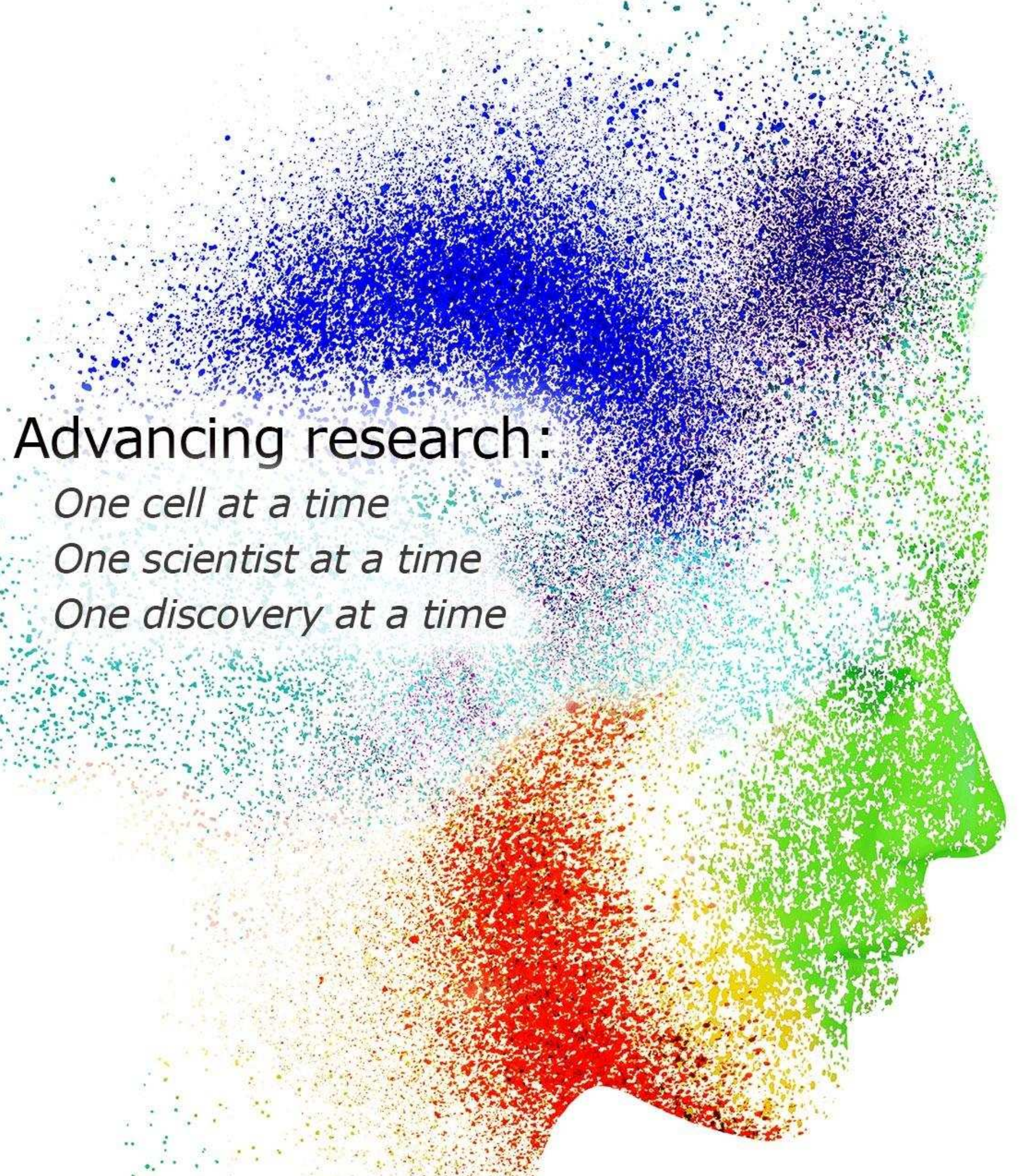

Proven solutions

that further science

BD Accurit ${ }^{\mathrm{TM}} \mathrm{C} 6$ Plus

BD FACSCelesta ${ }^{\text {TM }}$

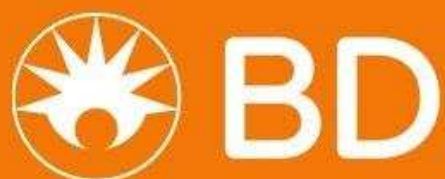

BD LSRFortessa ${ }^{\text {TM }}$

Discover more> 


\title{
Colon cancer associated transcript-1: a novel RNA expressed in malignant and pre-malignant human tissues
}

\author{
Aviram Nissan ${ }^{1}$, Alexander Stojadinovic ${ }^{2}$, Stella Mitrani-Rosenbaum ${ }^{3}$, David Halle ${ }^{1}$, Ronit Grinbaum ${ }^{1}$, \\ Marina Roistacher ${ }^{1}$, Andrea Bochem ${ }^{1}$, Baris Emre Dayanc ${ }^{4}$, Gerd Ritter ${ }^{5}$, Ismail Gomceli ${ }^{6}$, \\ Erdal Birol Bostanci ${ }^{6}$, Musa Akoglu ${ }^{6}$, Yao-Tseng Chen ${ }^{7}$, Lloyd John Old ${ }^{5}$ and Ali Osmay Gure ${ }^{4}$ \\ ${ }^{1}$ The Surgical Oncology Laboratory, Department of Surgery, Hadassah-Hebrew University Medical Center, Mount Scopus, Jerusalem, Israel \\ ${ }^{2}$ Division of Surgical Oncology, The Department of Surgery, Walter Reed Army Medical Center, Washington DC \\ ${ }^{3}$ Goldyne Savad Institute of Gene Therapy, Hadassah-Hebrew University Medical Center, Mount Scopus, Jerusalem, Israel \\ ${ }^{4}$ Department of Molecular Biology and Genetics, Bilkent University; Ankara, Turkey \\ ${ }^{5}$ The New York Branch of the Ludwig Institute for Cancer Research, Memorial Sloan Kettering Cancer Center, New York, NY \\ ${ }^{6}$ Department of Gastroenterological Surgery, Yuksek Ihtisas Training and Research Hospital, Ankara, Turkey \\ ${ }^{7}$ Department of Pathology, Weill-Cornell Medical Center, New York, NY
}

Early detection of colorectal cancer (CRC) is currently based on fecal occult blood testing (FOBT) and colonoscopy, both which can significantly reduce CRC-related mortality. However, FOBT has low-sensitivity and specificity, whereas colonoscopy is labor- and cost-intensive. Therefore, the discovery of novel biomarkers that can be used for improved CRC screening, diagnosis, staging and as targets for novel therapies is of utmost importance. To identify novel CRC biomarkers we utilized representational difference analysis (RDA) and characterized a colon cancer associated transcript (CCAT1), demonstrating consistently strong expression in adenocarcinoma of the colon, while being largely undetectable in normal human tissues $(p<000.1)$. CCAT1 levels in CRC are on average 235 -fold higher than those found in normal mucosa. Importantly, CCAT1 is strongly expressed in tissues representing the early phase of tumorigenesis: in adenomatous polyps and in tumor-proximal colonic epithelium, as well as in later stages of the disease (liver metastasis, for example). In CRC-associated lymph nodes, CCAT1 overexpression is detectable in all H\&E positive, and $40.0 \%$ of H\&E and immunohistochemistry negative lymph nodes, suggesting very high sensitivity. CCAT1 is also overexpressed in $40.0 \%$ of peripheral blood samples of patients with CRC but not in healthy controls. CCAT1 is therefore a highly specific and readily detectable marker for CRC and tumor-associated tissues.

Colorectal cancer (CRC) is a common disease affecting over a million people annually, worldwide. ${ }^{1}$ Novel cytotoxic agents alone or in combination with targeted systemic therapy significantly improve median survival in patients with advanced or metastatic CRC. These adjuvant therapeutic agents reduce the risk of disease-recurrence in patients who undergo complete resection of CRC, but are at high risk of disease relapse. Despite major advances in systemic therapy for CRC, nearly $50 \%$ of patients diagnosed with this common malignancy will recur and die of disease within 5 years of diagnosis and treatment with curative intent. ${ }^{2}$ To improve overall outcome of this disease, prevention and early detection through effective screening methods are imperative.

Current CRC screening and diagnosis is based mainly on fecal occult blood testing (FOBT) and fiber-optic colonoscopy, both which have demonstrated clinical utility and efficacy in early diagnosis and reduction of CRC-related mortality. ${ }^{3,4}$ Recently, stool-based DNA assays were developed for

Key words: colorectal cancer, non-coding RNA, biomarkers, minimal residual disease

Abbreviations: AMV: Avian Myeloblastosis Virus; Bp: base pair; CCAT1: colon cancer associated transcript-1; cDNA: complementary deoxy-ribonucleic acid; CEA: carcinoembryonic antigen; CRC: colorectal cancer; FOBT: fecal occult blood testing; H\&E: hematoxylin \& eosin; IRB: institutional review board; miRNA: microRNA; ORF: open reading frame; PBMC: peripheral blood mononuclear cells; RDA: representational difference analysis; RNA: ribonucleic acid; RQ: relative quantity; RT-PCR: real-time polymerase chain reaction; SNP: single nucleotide polymorphism; TCF7L2: transcription factor 7-like 2

Additional Supporting Information may be found in the online version of this article.

Grant sponsors: Hadassah-Hebrew University Medical Center, Ludwig Institute for Cancer Research

DOI: $10.1002 /$ ijc. 26170

History: Received 28 Jan 2011; Accepted 21 Mar 2011; Online 5 May 2011

Correspondence to: Aviram Nissan, M.D., Department of Surgery, Hadassah-Hebrew University Medical Center Mount Scopus, P.O.B. 24035, Jerusalem, 91240 Israel, Te.: +011-972-2-5844550, Fax: +011-972-2-5844028, E-mail: anissan@hadassah.org.il; or Ali Osmay Gure, Department of Molecular Biology and Genetics, SB-248, Bilkent University, Ankara, 06800 Turkey, Tel.: +90-312-290-2507,

Fax: +90-312-266-5097, E-mail: agure@bilkent.edu.tr 
CRC screening and tested in clinical trials. These screening assays are mainly based on genetic (somatic mutations) and epigenetic changes typical for CRC, ${ }^{5,6}$ but have thus far not achieved widespread population-based utilization.

Advancing screening and diagnostic capability in CRC will certainly require the identification of novel disease-specific bio-markers. Unfortunately, mass screening of CRC tissue samples using gene expression arrays have thus far failed to identify CRC-related molecular markers with sufficiently high sensitivity and specificity to be clinically useful for the purpose of screening and diagnosis. Novel CRC-specific molecules undetectable in normal tissues are not only critical for early diagnosis of CRC but may also serve as the fundamental basis for drug discovery and development of targeted therapeutics. The aim of the present study was to identify specific transcripts expressed uniquely in CRC that could be used for screening, diagnosis and staging of CRC.

\section{Methods}

\section{Cell lines and preparation of RNA}

The colon cancer cell lines HCT116, Colo205, HT-29 and SK-CO-10 were obtained from ATCC (Manassas, VA). Total RNA was obtained either by the guanidinium isothiocyanate/ $\mathrm{CsCl}$ gradient purification method or via the $\mathrm{TRIzol}^{\circledR}$ reagent (Sigma-Aldrich, St. Louis, MO). Normal tissue RNA was obtained from Clontech (Mountain view, CA).

\section{Representational difference analysis, cDNA cloning and rapid amplification of cDNA ends}

Representational difference analysis (RDA) was performed as described previously. ${ }^{7}$ Tester RNA was prepared from HT29 and RNA obtained from three normal (tumor-free) colon tissue specimens were pooled and used as driver RNA. Three rounds of amplification utilizing Tsp509I or DpnII as the restriction endonuclease were performed. Fragments generated after the third amplification cycles were isolated and sequenced. The partial length colon cancer associated transcript-1 (CCAT1) identified by RDA was used to screen a Colo20 5 cDNA library in the $\lambda$-ZAPII vector system (Stratagene, La Jolla, CA) by which the full length CCAT1 cDNA was obtained. For $5^{\prime}$ rapid amplification of $\mathrm{CDNA}$ ends (RACE), we used CCAT1-specific primers corresponding to bps 612-636 and 537-559, and the Marathon cDNA amplification kit, with adaptor-ligated colon cDNA as substrate (Clontech, Palo Alto, CA). Resulting bands were gel purified and cloned into pGEM-T vector (Promega, Madison, WI).

\section{Recombinant protein and polyclonal antibody generation}

Recombinant peptides corresponding to the three longest open reading frames (ORFs) of the CCAT1 gene (nt. 180293, 37aa; nt. 395-604, 69aa; nt. 1706-1855, 49aa) were cloned and purified as DHFR fusion proteins in the pQE system (Qiagen, Valencia, CA). Rabbit polyclonal antibodies against all three fusion proteins were produced by Covance (Denver, PA).

\section{Patients and tissue specimen collection}

Patients over the age of 18 years with histologically confirmed primary adenocarcinoma of the colon were offered participation in the study. Patients who received prior radiation or chemotherapy were ineligible for the study. The study protocol was approved by the Institutional Review Board (IRB, Helsinki Committee) of Hadassah-Hebrew University Medical Center, the Memorial Sloan Kettering Cancer Center-the New York Branch of the Ludwig Institute for Cancer Research, and the Yuksek Ihtisas Training and Research Hospital of Ankara. All samples were obtained from consenting study subjects undergoing surgical tumor resection who signed a written informed consent approved by their respective IRBs. All specimens underwent routine macroscopic and microscopic analysis by the pathologist. Tissues identified as CRC or adjacent normal tissues were then used for total RNA preparation. Colon carcinoma, adjacent normal tissue specimens and related tissues were provided by the New York Branch of the Ludwig Institute (tumors nos. 4-29), from the Hadassah-Hebrew University Medical Center (tumors nos. 96-861, lymph nodes and adenoma tissue), or from the Yuksek Ihtisas Hospital (CI, FC and SU).

\section{Blood samples}

Peripheral whole venous blood samples $(15 \mathrm{ml})$ were obtained at the Hadassah-Hebrew University Medical Center from 20 patients with CRC participating in the study, $24 \mathrm{hr}$ before the tumor was resected. Blood samples were transferred to the laboratory immediately and peripheral blood mononuclear cells (PBMC) were separated by the Ficoll gradient method and stored at $-70^{\circ} \mathrm{C}$.

\section{Surgical procedures}

All patients underwent standard surgical resection of adenocarcinoma of the colon including a wedge of associated mesentery containing the draining regional lymphatics. Immediately following removal of the surgical specimen (colon and mesentery), ex vivo lymphatic mapping was performed using 2-5 $\mathrm{ml}$ of Isosulfan blue dye (Patent Blue V, France), which was injected (ex vivo) sub-serosally in four quadrants around the tumor. The en-bloc resection specimen of tumor and lymph nodes was then evaluated on the back table and all blue nodes (sentinel lymph nodes, SLNs) from the mesentery were dissected from the specimen. One half of the SLN(s) was snap frozen in liquid nitrogen. A small portion $(\sim 25 \%$ of the entire tumor volume) of the primary tumor and a small sample $(0.5 \mathrm{~g})$ of normal mucosa were snap frozen similarly. Separate instruments were used for the SLN(s) and primary tumor dissection ex vivo to minimize tumor cell contamination of the node(s). The primary tumor specimen with attached mesentery and the remaining half of the $\operatorname{SLN}(\mathrm{s})$ were then submitted for standard histopathological examination including staining for cytokeratins as separately labeled specimens. Formalin-fixed paraffin-embedded sentinel 
nodes underwent serial step sectioning at 40-200 $\mu \mathrm{m}$ intervals. Four $4-\mu \mathrm{m}$ sections were stained with hematoxylin and eosin (H\&E) and two sections underwent immunhistochemsitry (IHC) using the avidin-biotin-peroxidase complex method according to a standardized commercially available pan-cytokeratin antibody cocktail protocol (Pan-keratin AE1/ AE3, CAM 5.2, 35bH11; Ventana Medical Systems, Tucson, AZ). Patients with synchronous liver metastasis underwent resection of the primary tumor with the draining lymph nodes and resection of liver metastasis during the same surgical procedure. SLN biopsy was not performed in this group of patients with liver metastases.

\section{RNA extraction from tissues}

Extraction of total RNA was performed for all samples (tissues and blood) by the TriReagent ${ }^{\circledR}$ method (Molecular Research Center, Cincinnati, OH) according to the manufacturer's instructions. Frozen samples were powdered in dry ice, homogenized using a Polytron homogenizer in TriReagent ${ }^{\circledR}$. RNA quality was confirmed by gel electrophoresis prior to the synthesis of cDNA.

\section{Quantitative reverse-transcriptase polymerase chain reaction}

One microgram of total RNA was used for reverse transcription with random primers in a $20 \mu \mathrm{l}$ reaction of which $2 \mu \mathrm{l}$ was used for polymerase chain reaction (PCR). All experiments were conducted in duplicate. Quantitative reverse-transcriptase polymerase chain reaction (qRT-PCR) was performed for 40 cycles (denaturation: $95^{\circ} \mathrm{C} \times 15 \mathrm{sec}$; annealing/extension: $\left.60^{\circ} \mathrm{C} \times 1 \mathrm{~min}\right)$ with the primers and TaqMan $^{\circledR}$ probe specific for either GAPDH (Human GAPD (GAPDH) Endogenous Control (VIC/MGB Probe, Primer Limited, 4326317E) or for $18 \mathrm{~S}$ (QuantumRNA ${ }^{\mathrm{TM}}$ Universal $18 S$ Internal StandardAM1718), obtained from Applied Biosystems, Foster City, CA.

For CCAT1 expression analysis, the primers and probe used were as follows:

- forward primer: 5'-TCACTGACAACATCGACTTTG AAG;

- reverse primer: 5'-GGAGAAAACGCTTAGCCATAC AG;

- probe: 6Fam-CTGGCCAGCCCTGCCACTTACCA-Tamra.

Relative quantification was done according to the manufacturer's instructions (Applied Biosystems, Foster City, CA; User Manual 2). Each sample was normalized according to its GAPDH or $18 \mathrm{~S}$ content and also against a calibrator which was either RNA obtained from normal lymph nodes, or normal colon (Ambion ${ }^{\circledR}$ Austin, TX). The relative quantity was determined according to the $\Delta \Delta \mathrm{CT}$ method using the

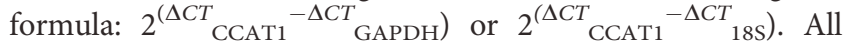
experiments were performed using an ABI Prism 7500 system (Applied Biosystems, Foster City, CA). To generate cali- brators for tumor cell RNA detection in blood, colon cancer cells were mixed in increasing concentrations with previously

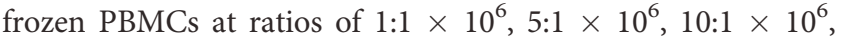
$50: 1 \times 10^{6}, 100: 1 \times 10^{6}$ and 500:1 $\times 10^{6}$. RNA was extracted from each mixture and qRT-PCR was performed for CCAT1 as described above.

\section{Statistical analysis}

Summary statistics were performed using established methods. Association between categorical factors was studied with Fisher's exact test or Chi-square test, as appropriate. Association between non-categorical (continuous) variables was performed using Students- $t$ test or ANOVA with Bonferroni post hoc test. SPSS statistical package version 13.0 (SPSS, Chicago, IL) was used for statistical analysis. Two sided $p$-value $\leq 0.05$ was considered significant.

\section{Results \\ Identification of transcripts with preferential expression in colon cancer}

RDA analysis with HT29 as tester and normal colon RNA as driver resulted in the identification of nine transcripts (Supplemental Table 1). Five of these (Bcl-x, MMP-7, ENC1, Met and MALAT1) were known be upregulated in cancer, ${ }^{8,9}$ three were reported to show ubiquitous expression (RNMT, LMAN1 and BPTF), ${ }^{10,11}$ and one was a novel transcript (CCAT1). By Northern analysis using the RDA-isolated fragments as probes, we confirmed Bcl-x, MMP-7 and ENC1 upregulation in 21 of 22, 7 of 22 and 6 of 22 colon cancer cell lines, respectively. In the same analysis, the CCAT1 RDA-probe hybridized to a single RNA species, that was upregulated in 10 of the 22 of the cell lines studied (Supplemental Fig. 1).

\section{Characterization of the CCAT1 transcript}

We used a CCAT1 RDA fragment to isolate the full-length CCAT1 cDNA from a HT29 cDNA library. Additional 5' RACE analysis with HT29 RNA identified three transcripts only one which had six additional nucleotides (Fig. 1). CCAT1 is identical to an incompletely characterized cDNA, AK125310, expressed in teratocarcinoma. ${ }^{12}$ Compared to AK125310, CCAT1 lacks 222 nucleotides at its $5^{\prime}$-end and has an additional 227 nucleotides at the $3^{\prime}$-end. The fulllength CCAT1 transcript is 2,628 nucleotides with two exons corresponding to nucleotides 1-288 and 289-2612. The gene has an unusually long intron of about $9 \mathrm{~kb}$ (Fig. 1). CCAT1 maps to chromosome $8 \mathrm{q} 24.21$ in close proximity to several mutational hot-spots associated with various cancers, including colorectal, prostate, breast and chronic lymphocytic leukemia (Supplemental Fig. 2).

\section{CCAT1 is putatively a non-coding RNA}

CCAT1 RNA contains three short ORFs. None of the putative translation products, however, have homology to any known or putative protein sequence. To test whether any of these transcripts were translated in colon cancer cell lines 


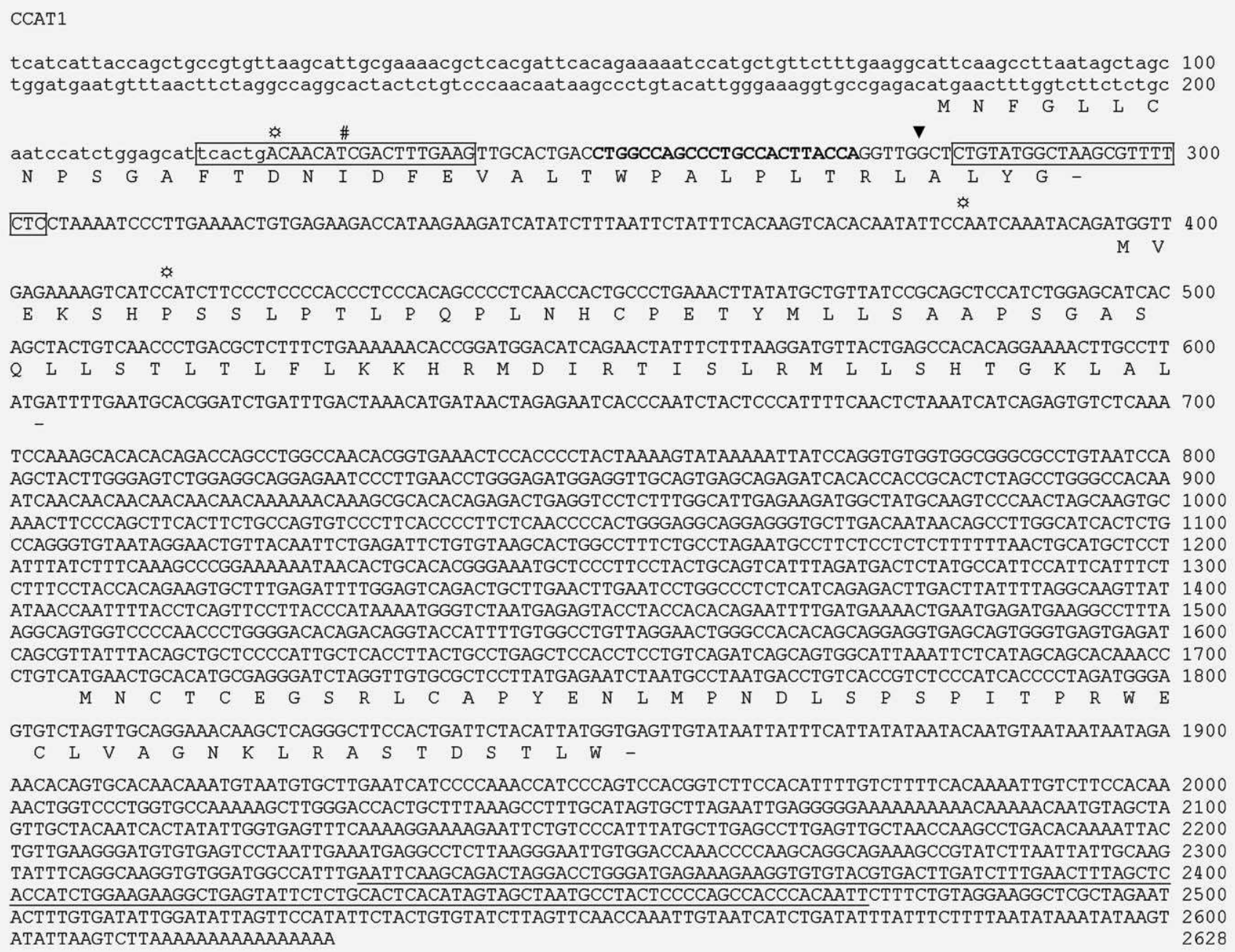
AGCTACTTGGGAGTCTGGAGGCAGGAGAATCCCTTGAACCTGGGAGATGGAGGTTGCAGTGAGCAGAGATCACACCACCGCACTCTAGCCTGGGCCACAA 900 ATCAACAACAACAACAACAACAAAAAACAAAGCGCACACAGAGACTGAGGTCCTCTTTGGCATTGAGAAGATGGCTATGCAAGTCCCAACTAGCAAGTGC 1000 AAACTTCCCAGCTTCACTTCTGCCAGTGTCCCTTCACCCCTTCTCAACCCCACTGGGAGGCAGGAGGGTGCTTGACAATAACAGCCTTGGCATCACTCTG 1100 CCAGGGTGTAATAGGAACTGTTACAATTCTGAGATTCTGTGTAAGCACTGGCCTTTCTGCCTAGAATGCCTTCTCCTCTCTTTTTTAACTGCATGCTCCT 1200 ATTTATCTTTCAAAGCCCGGAAAAAATAACACTGCACACGGGAAATGCTCCCTTCCTACTGCAGTCATTTAGATGACTCTATGCCATTCCATTCATTTCT 1300 CTTTCCTACCACAGAAGTGCTTTGAGATTTTGGAGTCAGACTGCTTGAACTTGAATCCTGGCCCTCTCATCAGAGACTTGACTTATTTTAGGCAAGTTAT 1400 ATAACCAATTTTACCTCAGTTCCTTACCCATAAAATGGGTCTAATGAGAGTACCTACCACACAGAATTTTGATGAAAACTGAATGAGATGAAGGCCTTTA 1500 AGGCAGTGGTCCCCAACCCTGGGGACACAGACAGGTACCATTTTGTGGCCTGTTAGGAACTGGGCCACACAGCAGGAGGTGAGCAGTGGGTGAGTGAGAT 1600 CAGCGTTATTTACAGCTGCTCCCCATTGCTCACCTTACTGCCTGAGCTCCACCTCCTGTCAGATCAGCAGTGGCATTAAATTCTCATAGCAGCACAAACC 1700 CTGTCATGAACTGCACATGCGAGGGATCTAGGTTGTGCGCTCCTTATGAGAATCTAATGCCTAATGACCTGTCACCGTCTCCCATCACCCCTAGATGGGA 1800 $\begin{array}{lllllllllllllllllllllllllllllllllll}M & N & C & T & C & E & G & S & R & L & C & A & P & Y & E & N & L & M & P & N & D & L & S & P & S & P & I & T & P & R & W & E\end{array}$

GTGTCTAGTTGCAGGAAACAAGCTCAGGGCTTCCACTGATTCTACATTATGGTGAGTTGTATAATTATTTCATTATATAATACAATGTAATAATAATAGA 1900 C L V V A

AACACAGTGCACAACAAATGTAATGTGCTTGAATCATCCCCAAACCATCCCAGTCCACGGTCTTCCACATTTTGTCTTTTCACAAAATTGTCTTCCACAA 2000 AACTGGTCCCTGGTGCCAAAAAGCTTGGGACCACTGCTTTAAAGCCTTTGCATAGTGCTTAGAATTGAGGGGGAAAAAAAAAACAAAAACAATGTAGCTA 2100 GTTGCTACAATCACTATATTGGTGAGTTTCAAAAGGAAAAGAATTCTGTCCCATTTATGCTTGAGCCTTGAGTTGCTAACCAAGCCTGACACAAAATTAC 2200 TGTTGAAGGGATGTGTGAGTCCTAATTGAAATGAGGCCTCTTAAGGGAATTGTGGACCAAACCCCAAGCAGGCAGAAAGCCGTATCTTAATTATTGCAAG 2300 TATTTCAGGCAAGGTGTGGATGGCCATTTGAATTCAAGCAGACTAGGACCTGGGATGAGAAAGAAGGTGTGTACGTGACTTGATCTTTGAACTTTAGCTC 2400 ACCATCTGGAAGAAGGCTGAGTATTCTCTGCACTCACATAGTAGCTAATGCCTACTCCCCAGCCACCCACAATTCTTTCTGTAGGAAGGCTCGCTAGAAT 2500 ACTTTGTGATATTGGATATTAGTTCCATATTCTACTGTGTATCTTAGTTCAACCAAATTGTAATCATCTGATATTTATTTCTTTTAATATAAATATAAGT 2600 ATATTAAGTCTTAAAAAAAAAAAAAAAA

Figure 1. CCAT1 nucleotide sequence and predicted translational products. Full length CCAT1 transcript and 3 ORFs are shown (GeneBank accession number: HM358356). Sequences corresponding to the RDA fragment are underlined. \#: $5^{\prime}$ end of the cDNA clone isolated from the Colo205 library. $5^{\prime}$ ends of three $5^{\prime}$-RACE transcripts. $\boldsymbol{\nabla}$ : exon/intron junction. Forward and reverse qRT-PCR primers are boxed and sequences corresponding to the probe are in bold font. Lower case letters correspond to additional sequences found in AK125310. AK125310 lacks 277 nts included in CCAT1 $3^{\prime}$ end.

expressing CCAT1 RNA, recombinant peptides corresponding to all three ORFs (nucleotides: 95-208, 37aa; 310-519, 69aa; 1,621-1,770, 49aa; Fig. 1) were cloned and purified as His-tagged DHFR fusion proteins using Ni-Cad chromatography. Polyclonal antibodies were raised against all three fusion proteins in rabbits. However, although polyclonal antibodies recognized bacterially expressed CCAT1 peptides, none recognized a specific protein in cell lysates prepared from colon carcinoma cell lines HT29, SW837 or LIM1215 (cell lines with detectable CCAT1 RNA expression) when tested by Western analysis (not shown). We, therefore, conclude that CCAT1 is very likely a non-coding RNA species.

\section{CCAT1 RNA is upregulated in colorectal cancer}

We quantified CCAT1 expression by qRT-PCR in a panel of 18 normal tissues and compared them to HCT116, a colo- rectal carcinoma cell line. HCT116 showed more than 100fold expression of CCAT1 over normal colon (Fig. 2). Among other normal tissues, the highest expression was observed in small intestine (33-fold relative increase in expression) and esophagus (14-fold).

We then tested CCAT1 expression in 28 colorectal tumor specimens and matching normal colon tissues. As shown in Figure 3, 24 of $28(85 \%)$ tissues showed more than 30 -fold upregulation of CCAT1 compared to normal colon $(p<$ $0.0001)$. Seventeen $(60 \%)$ had greater than 100 -fold relative increase in transcript expression $(p<0.0001)$. Upregulation of CCAT1 was also detected in tumor-adjacent normal colonic mucosa tissues, albeit to a lesser extent, with a mean upregulation of 12 -fold over normal colon tissue. Only one tumor-proximal tissue showed an upregulation of CCAT1 above 100 -fold, relative to normal colon (Fig. 3). CCAT1 
expression was found to decrease dramatically in normal mucosa obtained 5-40 cm proximal to three tumors (Supplemental Fig. 3). Only one tumor showed significant expression in normal mucosa $5 \mathrm{~cm}$ proximal to the tumor, whereas no significant CCAT1 expression was observed at sites obtained from $10 \mathrm{~cm}$ or beyond.

\section{CCAT1 expression in sentinel lymph nodes and peripheral} blood of colon cancer patients

To evaluate the potential role of CCAT1 in screening, diagnosis and staging of CRC, we tested CCAT1 expression in lymph node and peripheral blood samples from patients with CRC enrolled in the study. Six SLNs were obtained from four patients with tumor-positive staining by $\mathrm{H} \& \mathrm{E}$, and from five patients that were tumor-negative by $\mathrm{H} \& \mathrm{E}$ and immunohistochemistry

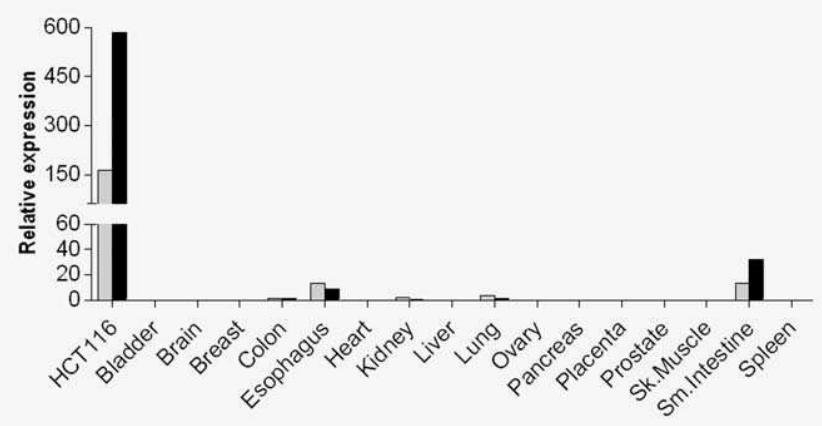

Figure 2. CCAT1 expression in normal tissues. Expression of the CCAT1 transcript as detected by qRT-PCR in a panel of normal tissues and in the colorectal cell line HCT116. Values correspond to fold increase relative to colon RNA when GAPDH (gray bars) or $18 \mathrm{~S}$ RNA (black bars) was used as control.
(IHC) for cytokeratin. All nine patients strongly expressed CCAT1 in their primary colon tumors. Compared to lymph nodes obtained from patients without malignancy and commercially available lymph node RNA, all lymph nodes from tumor positive and two from tumor negative patients showed upregulation of CCAT1 by more than 100-fold (Fig. 4).

To quantify CCAT1 RNA in the peripheral blood of patients with CRC, we generated calibrator RNAs by mixing HT-29 cells with blood mononuclear cells (PBMC) obtained from peripheral whole venous blood, at ratios ranging from $1: 10^{6}$ to $500: 10^{6}$. Total RNA from these cell mixtures was then tested for CCAT1 expression by qRT-PCR. Amplification results showed a linear correlation between CCAT1 levels as determined by qRT-PCR and tumor cell to PBMC ratios (Supplemental Fig. 4). Total RNA from PBMCs were obtained from eight healthy volunteers and 16 patients with $\mathrm{CRC}$, and amplification results were compared to those obtained from the calibration experiment. CCAT1 expression in healthy volunteer PBMCs ranged from 0.8- to 4.5-fold compared to that obtained for the $1: 10^{6}$ dilution, while those obtained from patients with CRC were between 0.1 and 1700-fold (Fig. 5). PBMCs from 7 of 16 (45\%) patients showed CCAT1 expression of more than 10-fold over that obtained from the highest dilution of calibrator RNA, while 1 showed 1,700-fold upregulation of CCAT1 (Fig. 5).

\section{CCAT1 expression in adenomatous polyps} and metastases from CRC

We next tested CCAT1 expression by qRT-PCR in tissues obtained from seven patients with adenomatous polyps and compared results to those obtained from four different colonic mucosa samples from patients who underwent surgery for non-cancer related causes, as well as to commercially

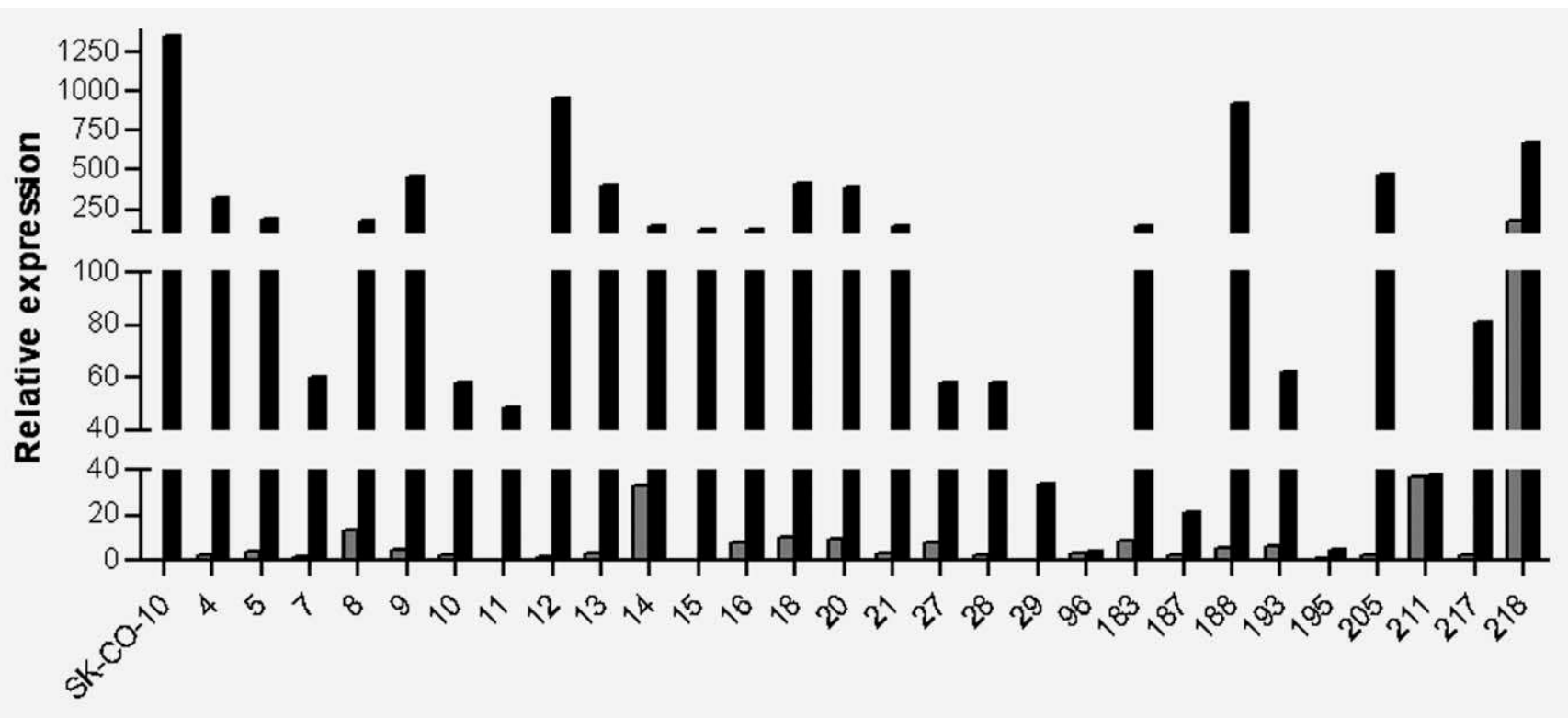

Figure 3. CCAT1 expression in colon carcinomas and adjacent normal tissues. qRT-PCR analysis of CCAT1 transcript levels in 28 colon carcinomas (black) and adjacent normal tissue (gray) is shown. Values correspond to fold increase relative to colon RNA. 

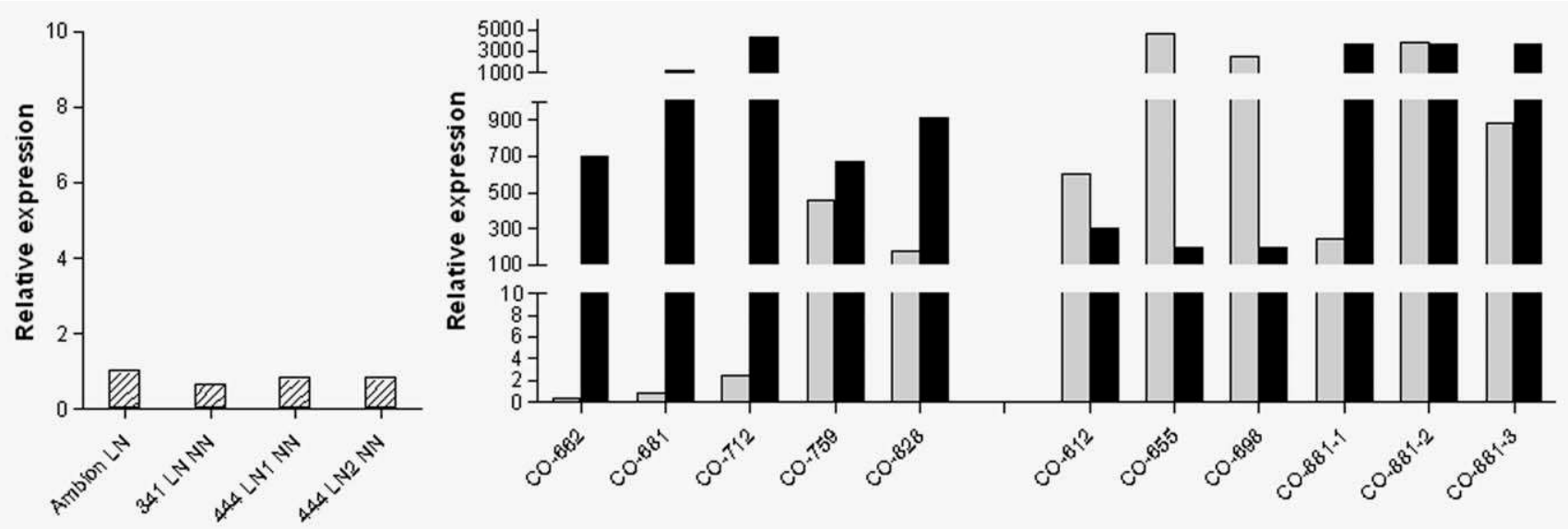

Figure 4. CCAT1 expression in normal and tumor-associated lymph node tissues. CCAT1 expression in commercial lymph node RNA (Ambion NL) and three normal lymph node samples obtained from two healthy controls (341 LN NN and 444 LN NN) are shown (a). CAAT1 expression in sentinel lymph node tissue obtained from five patients with negative lymph-nodes by H\&E and immunohistochemistry for cytokertin staining (CO-662, -681, -712, -759, -828) and from those with positive lymph nodes (C0-612, -655, -698, -881 (three samples)) are shown in gray (b). CCAT1 expression in tumors from the same individuals is shown in black. Values correspond to fold increase relative to normal lymph node RNA (Ambion).

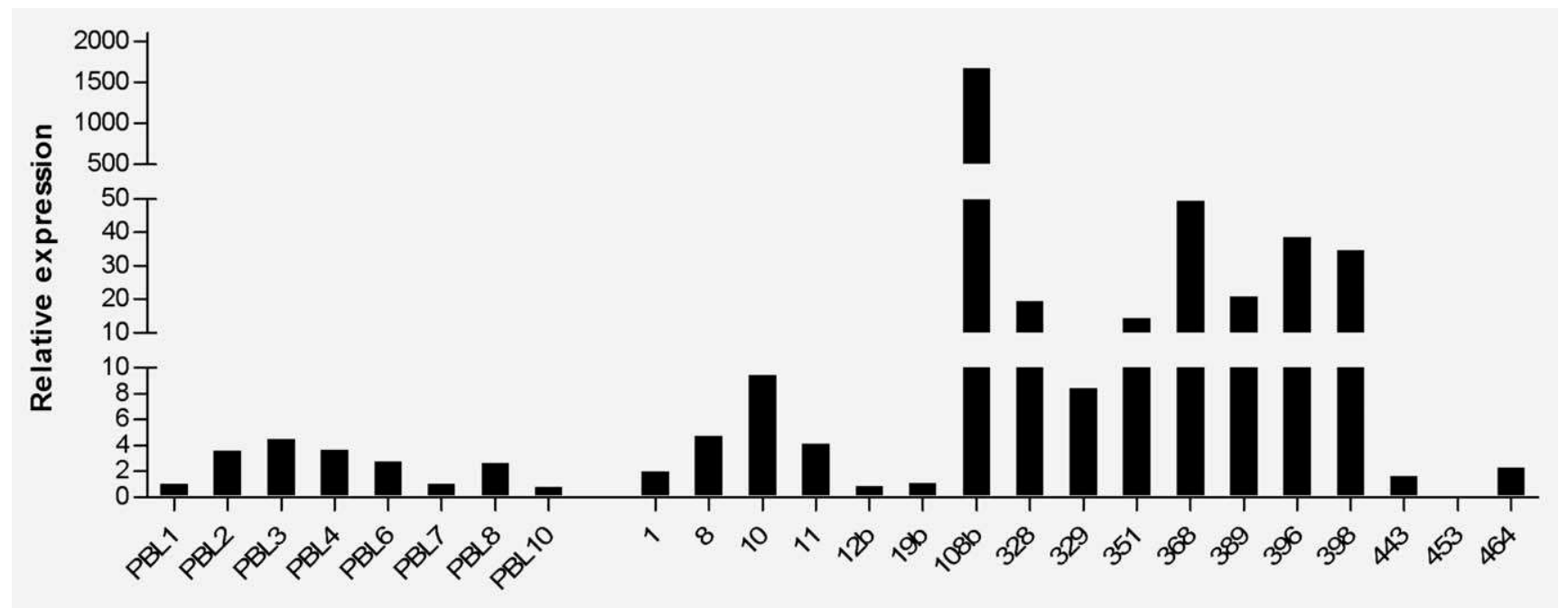

Figure 5. CCAT1 expression in PBMC samples of colon cancer patients. CCAT1 transcript levels were determined by qRT-PCR in PBMCS of control patients (NN1, -2, PBL1-10) and colon cancer patients (1-464). Values correspond to fold increase relative to that of the cell mixture with HT29 to normal PBMC ratio of $1: 10^{6}$ (see Supplemental Fig. 4).

available colon RNA. CCAT1 was upregulated more than 100 -fold in all adenomatous polyps, when compared to normal colon tissue (mean upregulation: 155-fold; $p<0.0001$ ); Figure 6. CCAT1 was also significantly upregulated in metastatic tissue. RNA obtained from five patients with CRC metastases to either the liver or the peritoneal cavity showed CCAT1 upregulation of more than 100-fold compared to normal colonic tissue, with four showing upregulation of over 450-fold (Fig. 6).

\section{Discussion}

CCAT-1 is a unique RNA upregulated in CRC compared to healthy tissues with very high specificity and sensitivity.
CCAT1 upregulation is less pronounced when tumor-neighboring tissue is compared to normal colonic tissue, suggesting a "field effect." Despite the fact that CCAT1 undergoes splicing and poly-adenylation, no translational products are detectable in colon cancer cell lines in our studies strongly suggesting that CCAT1 is a non-coding RNA.

The well established biological model of sequential progression of colorectal tumorigenesis provides an excellent system to critically assess biomarkers potentially useful for screening and early detection of CRC. The development of CRC generally occurs in a staged progression from normal mucosa, to adenoma, carcinoma in situ and finally carcinoma. In pre-malignant tissue (adenomatous polyps), we 


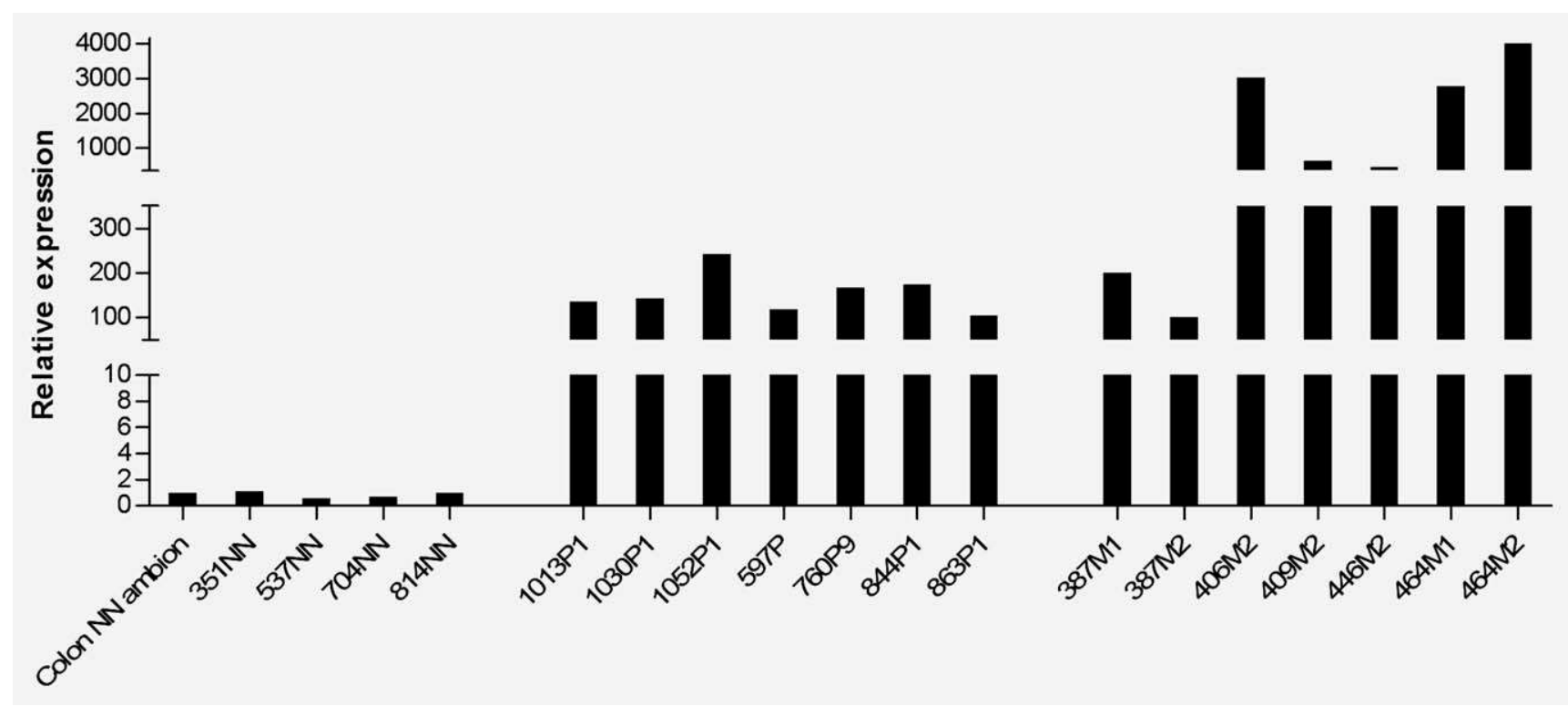

Figure 6. CCAT1 expression in adenomatous polyps and CRC metastases. CCAT1 expression in healthy control RNA (Colon NN Ambion, 351NN, 537NN, 704NN, 814NN) and in RNA from adenomatous polyps from 7 patients (1013P1, 1030P1, 1052P1, 597P, 760P9, 844P1, 863P1) and in CRC metastases to liver and peritoneal surface from 5 patients (387, 406, 409, 446, 464; M1 and M2). Values correspond to fold increase relative to normal colon RNA (Ambion).

could demonstrate consistently high CCAT1 expression suggesting a putative role for this non-coding RNA in early colorectal carcinogenesis. Importantly, we observed CCAT1 expression in regional nodal and distant liver metastasis, as well as in blood samples of patients with CRC, suggesting that testing for CCAT1 expression can detect small amounts of tumor cells. This level of detection extends to both metastatic tissue as well as circulating CRC tumor cells. Although larger studies are necessary to establish a role for CCAT1 in CRC diagnosis, the extremely high tumor to normal tissue ratios of its transcript suggests an unprecedented specificity for cancer with very high sensitivity. The overexpression of CCAT1 in all stages of the well established adenoma-to-carcinoma sequence singles it out as a potential biomarker for screening, diagnosis, staging and perhaps overall prognostication of CRC.

Currently, the only generally accepted biomarkers in general clinical use for patients with CRC are serum CEA and CA-19-9, neither of which is sufficiently specific or sensitive for use in disease screening or diagnosis. ${ }^{13,14} \mathrm{~A}$ large number of genetic and epigenetic alterations have been studied as potential biomarkers for screening, staging or determining the prognosis in various common epithelial cancers, including CRC. ${ }^{15}$ For example, a comprehensive screen for gene alterations revealed the presence of about 93 driver mutations frequently occurring in CRC, of which only 14 on average are present in any given tumor from a given individual. ${ }^{16}$ Changes in DNA methylation patterns of specific regions have also been reported as common molecular alterations in cancer. Over 100 genes undergoing aberrant DNA methylation have been defined in CRC. ${ }^{17,18}$ Integrated approaches consisting of both genetic and epigenetic analysis appear to have improved sensitivity and specificity for defining subtypes of CRC of varying biology. ${ }^{19}$ Despite these advances, the scope and complexity of these alterations require the development of an assay system whose complexity would make it difficult to introduce into general clinical use.

The CRC transcriptome has also been studied. In one study, about $0.48 \%$ of protein coding gene transcripts were upregulated 4- to 10 -fold relative to normal colonic tissue, ${ }^{20}$ while another transcriptomic study identified one transcript that was upregulated as much as 50 -fold in CRC over normal tissues. ${ }^{21}$ Importantly, the innate design of most highthroughput assays results in the complete exclusion of potentially useful differentiating, non-coding transcripts from the analyses. However, compared to mRNAs, there might be up to five times more non-coding RNA species transcribed from the genome, and more than half of these have been reported to show tissue/cell line specificity. ${ }^{22}$ It is, therefore, not surprising that two of the nine transcripts identified in this study correspond to long non-coding RNAs. There are only a few examples of heretofore described non-coding RNAs that are highly expressed in neoplastic but not in normal tissues. Some of these non-coding RNAs, like $\mathrm{H}-19$, relate to loss of imprinting in cancer. H-19 is highly expressed in tumors of the bladder, endometrium, ovary and testis ${ }^{23}$; as well as in liver metastasis originating from various epithelial cancer types including CRC. ${ }^{24}$

Genome-wide association studies have revealed a concentration of single nucleotide polymorphisms (SNPs) on $8 \mathrm{q} 24.21$ that are strongly associated with various cancers, ${ }^{25-28}$ clustered within position $128.0 \mathrm{Mb}$ to $128.6 \mathrm{Mb}$, flanking the 
CCAT1 gene (Supplemental Fig. 2). A SNP strongly associated with chronic lymphocytic leukemia, rs2456449, is located only $3 \mathrm{~kb}$ from the $3^{\prime}$ end of CCAT $1 .{ }^{28}$ No specific transcript has been identified from this "hot-spot" thus far, with the exception of CCAT1. This region of interest has been shown to interact with MYC, ${ }^{29,30}$ and the SNP rs6983267 at position $128.4 \mathrm{Mb}$, which influences MYC transcription. $^{31}$ Recently, another non-coding RNA, HOTAIR, has been shown to directly alter chromatin modifications resulting in upregulation of genes related to tumor invasiveness and metastasis. ${ }^{32}$ It will be important to determine whether CCAT1 transcription has a direct effect on carcinogenesis, or whether this non-coding RNA is a by-product of the epigenetic alterations known to take place in its vicinity. ${ }^{30}$

The fact that CCAT1 is expressed at very high levels even in pathologically staged apparently node-negative (N0) patients with colorectal carcinoma suggests that these patients might have low-volume, nodal metastases that are below the threshold of detection for nodal staging techniques currently utilized in standard practice. It is well recognized that the majority $(70-75 \%)$ of conventionally staged N0 patients will not develop recurrence of CRC, based on an existing staging and treatment decision paradigm of bivariate categorical nodal staging (positive or negative). The discovery of CCAT1 comes at a time of an emerging paradigm in nodal staging of CRC, one of quantifying tumor cell volume using techniques such as qRT-PCR, in order to stratify risk of disease recurrence with increasing, clinically meaningful, accuracy. The exact biomarker-specific parameters of nodal tumor volume correlating with recurrence risk remain to be defined, and represents an unmet need in CRC. Further studies will help define and validate a critical positive predictive quantitative threshold of tumor CCAT1 in an effort to prospectively define homogeneous sub-populations of pathologically staged pN0 (node-negative) patients who are at significant risk of recurrence that may benefit from targeted therapies.

\section{References}

1. Jemal A, Siegel R, Ward E, Hao Y, Xu J, Thun MJ. Cancer statistics, 2009. CA Cancer J Clin 2009;59:225-49.

2. Edwards BK, Ward E, Kohler BA, Eheman C, Zauber AG, Anderson RN, Jemal A, Schymura MJ, Lansdorp-Vogelaar I, Seeff LC, van Ballegooijen M, Goede SL, et al. Annual report to the nation on the status of cancer, 1975-2006, featuring colorectal cancer trends and impact of interventions (risk factors, screening, and treatment) to reduce future rates. Cancer 2010;116: 544-72.

3. Mandel JS, Bond JH, Church TR, Snover DC, Bradley GM, Schuman LM, Ederer F. Reducing mortality from colorectal cancer by screening for fecal occult blood. Minnesota Colon Cancer Control Study. N Engl J Med 1993;328:1365-71.

4. Kronborg O, Fenger C, Olsen J, Jorgensen OD, Sondergaard O. Randomised study of screening for colorectal cancer with faecaloccult-blood test. Lancet 1996;348:1467-71.

5. Kim MS, Lee J, Sidransky D. DNA methylation markers in colorectal cancer. Cancer Metastasis Rev 2010;29:181-206.

6. Potack J, Itzkowitz SH. Practical advances in stool screening for colorectal cancer. $J$ Natl Compr Cancer Netw 2010;8:81-92.

7. Gure AO, Stockert E, Arden KC, Boyer AD, Viars CS, Scanlan MJ, Old LJ, Chen YT. CT10: a new cancer-testis (CT) antigen homologous to CT7 and the MAGE family, identified by representational-difference analysis. Int $J$ Cancer 2000;85:726-32.

8. Sulkowski S, Kanczuga-Koda L, Koda M, Wincewicz A, Sulkowska M. Insulin-like growth factor-I receptor correlates with connexin 26 and $\mathrm{Bcl}-\mathrm{xL}$ expression in human colorectal cancer. Ann NY Acad Sci 2006;1090:265-75.

9. Umeki K, Shiota G, Kawasaki H. Clinical significance of c-met oncogene alterations in human colorectal cancer. Oncology 1999; 56:314-21.

10. Tsukamoto T, Shibagaki Y, Niikura Y, Mizumoto K. Cloning and characterization of three human cDNAs encoding mRNA (guanine-7-)-methyltransferase, an mRNA cap methylase. Biochem Biophys Res Commun 1998;251:27-34.

11. Neve EP, Svensson K, Fuxe J, Pettersson RF. VIPL, a VIP36-like membrane protein with a putative function in the export of glycoproteins from the endoplasmic reticulum. Exp Cell Res 2003;288:70-83.

12. Ota T, Suzuki Y, Nishikawa T, Otsuki T, Sugiyama T, Irie R, Wakamatsu A, Hayashi K, Sato H, Nagai K, Kimura K, Makita $\mathrm{H}$, et al. Complete sequencing and characterization of 21,243 full-length human cDNAs. Nat Genet 2004;36:40-5.

13. Ariel I, Ayesh S, Perlman EJ, Pizov G, Tanos V, Schneider T, Erdmann VA, Podeh D, Komitowski D, Quasem AS, de Groot N, Hochberg A. The product of the imprinted $\mathrm{H} 19$ gene is an oncofetal RNA. Mol Pathol 1997;50:34-44.

14. Ariel I, Lustig O, Schneider T, Pizov G, Sappir M, De-Groot N, Hochberg A. The imprinted $\mathrm{H} 19$ gene as a tumor marker in bladder carcinoma. Urology 1995;45:335-8.

15. Ahlquist DA. Molecular detection of colorectal neoplasia. Gastroenterology 2010; 138:2127-39.

16. Wood LD, Parsons DW, Jones S, Lin J, Sjoblom T, Leary RJ, Shen D, Boca SM,
Barber T, Ptak J, Silliman N, Szabo S, et al. The genomic landscapes of human breast and colorectal cancers. Science 2007;318: 1108-13.

17. Jubb AM, Bell SM, Quirke P. Methylation and colorectal cancer. J Pathol 2001;195: 111-34.

18. Zitt M, Zitt M, Muller HM. DNA methylation in colorectal cancer-impact on screening and therapy monitoring modalities? Dis Markers 2007;23:51-71.

19. Shen L, Toyota M, Kondo Y, Lin E, Zhang L, Guo Y, Hernandez NS, Chen X, Ahmed S, Konishi K, Hamilton SR, Issa JP. Integrated genetic and epigenetic analysis identifies three different subclasses of colon cancer. Proc Natl Acad Sci USA 2007;104: 18654-9.

20. Notterman DA, Alon U, Sierk AJ, Levine AJ. Transcriptional gene expression profiles of colorectal adenoma, adenocarcinoma, and normal tissue examined by oligonucleotide arrays. Cancer Res 2001;61:3124-30.

21. Sabates-Bellver J, Van der Flier LG, de Palo M, Cattaneo E, Maake C, Rehrauer H, Laczko E, Kurowski MA, Bujnicki JM, Menigatti M, Luz J, Ranalli TV, et al. Transcriptome profile of human colorectal adenomas. Mol Cancer Res 2007;5:1263-75.

22. Cheng J, Kapranov P, Drenkow J, Dike S, Brubaker S, Patel S, Long J, Stern D, Tammana H, Helt G, Sementchenko V, Piccolboni A, et al. Transcriptional maps of 10 human chromosomes at 5-nucleotide resolution. Science 2005;308:1149-54.

23. Ohlsson R. Loss of IGF2 imprinting: mechanisms and consequences. Novartis Found Symp 2004;262:108-21; discussion 21-4, 265-8. 
24. Fellig Y, Ariel I, Ohana P, Schachter P, Sinelnikov I, Birman T, Ayesh S, Schneider T, de Groot N, Czerniak A, Hochberg A. H19 expression in hepatic metastases from a range of human carcinomas. J Clin Pathol 2005;58:1064-8.

25. Murabito JM, Rosenberg CL, Finger D, Kreger BE, Levy D, Splansky GL, Antman $\mathrm{K}$, Hwang SJ. A genome-wide association study of breast and prostate cancer in the NHLBI's Framingham Heart Study. BMC Med Genet 2007;8 (Suppl 1):S6.

26. Thomas G, Jacobs KB, Yeager M, Kraft P, Wacholder S, Orr N, Yu K, Chatterjee N, Welch R, Hutchinson A, Crenshaw A, Cancel-Tassin G, et al. Multiple loci identified in a genome-wide association study of prostate cancer. Nat Genet 2008; 40:310-15.

27. Tenesa A, Farrington SM, Prendergast JG, Porteous ME, Walker M, Haq N,
Barnetson RA, Theodoratou E, Cetnarskyj R, Cartwright N, Semple C, Clark AJ, et al. Genome-wide association scan identifies a colorectal cancer susceptibility locus on $11 \mathrm{q} 23$ and replicates risk loci at $8 \mathrm{q} 24$ and 18q21. Nat Genet 2008;40:631-7.

28. Crowther-Swanepoel D, Broderick P, Di Bernardo MC, Dobbins SE, Torres M, Mansouri M, Ruiz-Ponte C, Enjuanes A, Rosenquist R, Carracedo A, Jurlander J, Campo E, et al. Common variants at 2q37.3, 8q24.21, 15q21.3 and 16q24.1 influence chronic lymphocytic leukemia risk. Nat Genet 2010;42:132-6.

29. Pomerantz MM, Ahmadiyeh N, Jia L, Herman P, Verzi MP, Doddapaneni H, Beckwith CA, Chan JA, Hills A, Davis M, Yao K, Kehoe SM, et al. The 8q24 cancer risk variant rs6983267 shows long-range interaction with MYC in colorectal cancer. Nat Genet 2009;41:882-4.
30. Ahmadiyeh N, Pomerantz MM, Grisanzio C, Herman P, Jia L, Almendro V, He HH, Brown M, Liu XS, Davis M, Caswell JL, Beckwith CA, et al. 8q24 prostate, breast, and colon cancer risk loci show tissuespecific long-range interaction with MYC. Proc Natl Acad Sci USA 2010;107: 9742-6.

31. Sotelo J, Esposito D, Duhagon MA, Banfield K, Mehalko J, Liao H, Stephens RM, Harris TJ, Munroe DJ, Wu X. Longrange enhancers on $8 \mathrm{q} 24$ regulate c-Myc. Proc Natl Acad Sci USA 2010;107: 3001-5.

32. Gupta RA, Shah N, Wang KC, Kim J, Horlings HM, Wong DJ, Tsai MC, Hung T, Argani P, Rinn JL, Wang Y, Brzoska P, et al. Long non-coding RNA HOTAIR reprograms chromatin state to promote cancer metastasis. Nature 2010;464: 1071-6. 